\title{
Emergence of canine ocular thelaziosis caused by Thelazia callipaeda in southern Switzerland
}

\author{
F. Malacrida ${ }^{\text {a }}$, D. Hegglin ${ }^{\text {a }}$, L. Bacciarini ${ }^{b}$, D. Otranto ${ }^{\text {c }}$, F. Nägeli ${ }^{\text {d }}$, \\ C. Nägeli ${ }^{d}$, C. Bernasconi ${ }^{d}$, U. Scheu $^{d}$, A. Balli $^{\mathrm{d}}$, M. Marenco ${ }^{\mathrm{d}}$, \\ L. Togni ${ }^{\mathrm{d}}$, P. Deplazes ${ }^{\mathrm{a}}$, M. Schnyder ${ }^{\mathrm{a}, *}$ \\ ${ }^{a}$ Institute of Parasitology, Vetsuisse Faculty, University of Zurich, Winterthurerstr. 266a, CH-8057 Zurich, Switzerland \\ ${ }^{\mathrm{b}}$ Cantonal Veterinary Office, Via Dogana 16, CH-6500 Bellinzona, Switzerland \\ ${ }^{\mathrm{c}}$ Department of Veterinary Public Health, Faculty of Veterinary Medicine, University of Bari, Str. Prov. Casamassima Km 3, \\ I-70010 Valenzano, Bari, Italy \\ ${ }^{\mathrm{d}}$ Veterinary Practitioners in the Canton of Ticino, Switzerland
}

Received 19 May 2008; received in revised form 18 July 2008; accepted 25 July 2008

\begin{abstract}
In Europe, Thelazia callipaeda has been reported in Italy and France in the eyes of dogs, cats and foxes and, recently, also in humans. In southern Switzerland (Ticino), the first case of T. callipaeda in a dog was detected in 2000 and because of an increasing number of dog thelaziosis, a survey in veterinary practices was carried out. A total of 106 Thelazia-positive dogs from a retrospective analysis and from ongoing cases between 2005 and 2007 as well as five positive cats were reported. For a crosssectional study, 529 randomly selected dogs (from six veterinary practices), to which anaesthesia was given for other medical reasons, were additionally checked for the presence of adult specimens of Thelazia in 2006: 28 dogs were found positive indicating an overall prevalence of $5.3 \%$. Thelazia-infection was furthermore diagnosed in 7 of 126 foxes (5.6\%) shot in Ticino in winter 2005-2006. Affected foxes, dogs and cats originated from the same regions up to $863 \mathrm{~m}$ of altitude. The cats and 57.9\% (55/95) of the infected dogs had never crossed the Swiss border. Collected nematodes were morphologically identified as T. callipaeda, and this diagnosis was confirmed by the analysis of a part of the sequence of the mitochondrial cytochrome $c$ oxidase subunit 1 gene (cox1), revealing haplotype 1, the only one so far found in Europe. Animals harboured 1-23 eye worms. The most common symptoms were conjunctivitis and epiphora, while keratitis was present only in a low number of animals. Young and small sized dogs were significantly less involved than large animals and over 3 years of age. The results indicate that thelaziosis is endemic in that area.
\end{abstract}

(C) 2008 Elsevier B.V. All rights reserved.

Keywords: Thelazia callipaeda; Nematode; Dog; Fox; Cat; Switzerland; Europe

\footnotetext{
* Corresponding author. Tel.: +41 446358501 ; fax: +41446358907.

E-mail address: manuela.schnyder@access.uzh.ch (M. Schnyder).
}

\section{Introduction}

Canine thelaziosis in Europe is caused by Thelazia callipaeda which also infects cats, foxes and rabbits (Otranto and Traversa, 2005). The occurrence of $T$. callipaeda has been described in dogs in northern Italy (Rossi and Bertaglia, 1989), later in dogs, cats and foxes in southern Italy (Otranto et al., 2003) and in dogs in 
France (Dorchies et al., 2007). Furthermore, four cases in humans have been reported in the European endemic area (Otranto and Dutto, 2008). Adult and larval stages can cause mild to severe irritation leading to lacrimation, epiphora, conjunctivitis, keratitis and/or corneal ulcers in dogs (Anderson, 2000; Otranto and Traversa, 2005).

Recent investigations identified Phortica variegata (Diptera, Drosophilidae) as a potential vector and intermediate host of $T$. callipaeda under experimental and natural conditions (Otranto et al., 2005a, 2006b). An ecological niche model suggested that the climate of large areas of Europe could be suitable habitats for this vector (Otranto et al., 2006a) and thus are potentially endemic for T. callipaeda.

Over the past few years, anecdotal reports on the occurrence of canine thelaziosis have been increasingly recorded in Ticino (southern Switzerland). Therefore, the aim of this study was to assess the endemic presence of $T$. callipaeda in this region.

\section{Materials and methods}

\subsection{Study area and data collection}

The study was carried out in an area of about $1300 \mathrm{~km}^{2}$ (Ticino canton) and in the neighbouring Mesolcina valley (Grisons canton) in the southern part of Switzerland. The altitude of the survey area ranged from 200 to over $3000 \mathrm{~m}$ above sea level (a.s.l.).

A retrospective analysis on the occurrence of canine thelaziosis was carried out for the time before 2005 by examining the clinical records of 16 of $22(72.7 \%)$ small animal veterinary practices in the canton of Ticino. Ongoing diagnosed cases were reported by the same practitioners from May 2005 to July 2006 and during the winter of 2006-2007. Additionally, from July to September 2006, in a cross-sectional study, 529 dogs from six veterinary clinical practices (i.e., five in the Ticino canton and one in the Mesolcina valley) were examined for canine thelaziosis by deepened inspection behind the third eye lid and anamnestic data were collected. These dogs underwent clinical examination or were anaesthetized for other reasons than eye problems.

The Ticino cantonal veterinary office provided data about dog population (number of animals, gender, age, breed and size). This information is registered for every Swiss dog since January 2007, according to the instructions given by the databank for the registration of pet animals in Switzerland (i.e., ANIS, Animal Identity Service). A high number of dogs were not classified concerning breed or size; this was due to the fact that crossbreed dogs could not be attributed to a category. However, we assumed that this should not influence data elaboration, since crossbreed dogs may be allocated to the four categories in a proportional manner.

Additionally, from December 2005 to January 2006, 126 foxes shot in the canton (Fig. 2B) were examined for the presence of eye worms.

\subsection{Parasite collection and identification}

Eye worms were extracted from the patient's eyes after local anaesthesia by tweezers, microscopically examined and morphologically identified according to Bhaibulaya et al. (1970). In order to confirm the morphological identification and to analyse the haplotype sequence, eight specimens were molecularly processed via the specific amplification by PCR of a partial sequence of the mitochondrial cytochrome $c$ oxidase subunit 1 gene (cox1, $689 \mathrm{bp}$ ) as previously described (Otranto et al., 2005b).

\subsection{Statistical analysis}

Data were statistically examined using SPSS-PC version 10.0 (Norusis, 1986). Regional prevalences were tested for significant differences by Fisher's exact test. $p$-Values are given two-tailed if not stated otherwise. A stepwise backward logistic regression procedure was used to test the effect of 'gender', 'age class', 'breed size', 'regional location' and 'sojourn in Italy' on the infection risk for dogs from the crosssectional study.

\section{Results and discussion}

A total of 106 Thelazia-positive dogs from the retrospective analysis (31) and the active search (75) were investigated in collaboration with the veterinarians and animal owners. In addition, five cats with thelaziosis were registered in this period, but no crosssectional study was undertaken for cats. The locations of Thelazia-positive dogs and cats are shown in Fig. 1A and B, respectively. Eye worms were collected from 74 out of 146 Thelazia-infected animals (from $63 \mathrm{dogs}, 4$ cats and 7 foxes) and in total 291 specimens of Thelazia sp. (259 from dogs, 5 from cats and 27 from foxes) were morphologically identified as $T$. callipaeda. Furthermore, the cox 1 sequences of eight specimens (from four dogs, three foxes and one cat originating from different regions in Ticino) were identical to the 


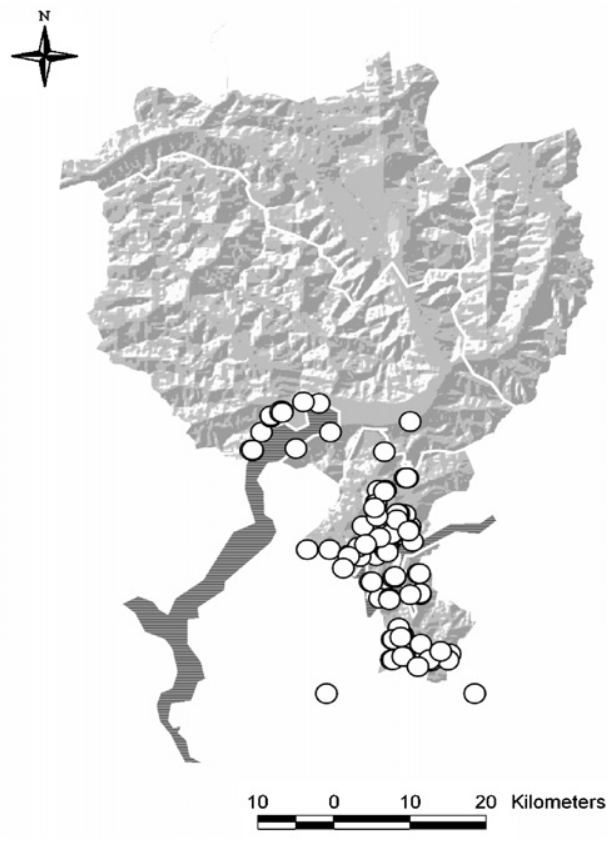

A: dogs $(n=106)$

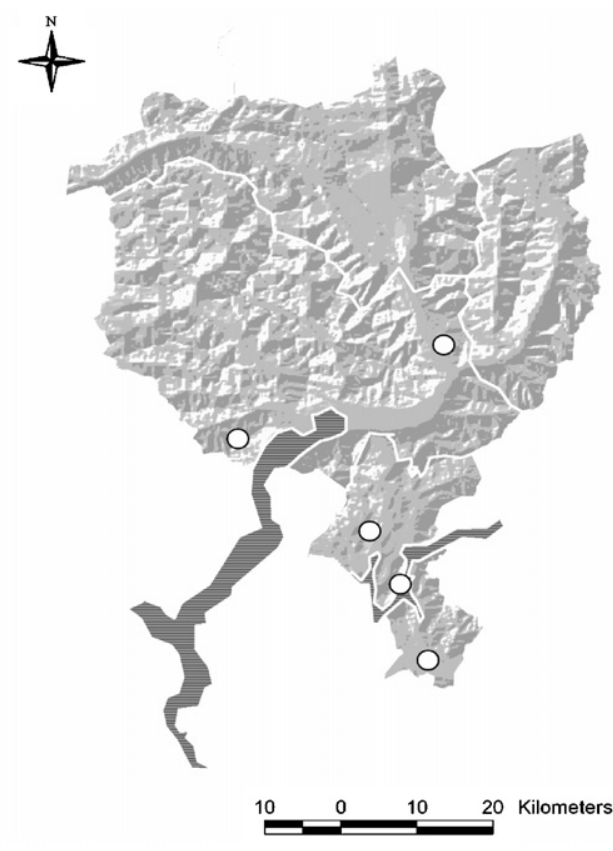

B: cats $(n=5)$

*: 2 dog owners with domicile

Fig. 1. Distribution of Thelazia callipaeda in southern Switzerland: (A) localisation of 31 cases from a retrospective analysis and of 75 ongoing cases diagnosed in 16 veterinary clinics and (B) localisation of 5 positive cats during the same period.

sequence representing haplotype 1 , confirming the low genetic variability of this nematode in Europe (Otranto et al., 2005b).

The retrospective analysis of eye worm infections revealed the first two cases of T. callipaeda in dogs in 2000. From 2001 to 2003, between 2 and 5 cases were recorded per annum while 20 cases were recorded in 2004. For the cross-sectional study, performed in dogs only, the investigated area was divided into four parts: northern, central and southern Ticino and Mesolcina valley. Fig. 2A presents the locations of 28 Thelaziapositive out of 529 dogs without medical history or suspected eye worms, showing an overall prevalence of $5.3 \%$ in the investigated area.

The location of 126 hunted foxes is shown in Fig. 2B. Out of 126 foxes examined 7 (5.6\%) were positive. Dogs and foxes shot in the northern part were all negative but differences between regions were not significant.

This study demonstrates the occurrence of autochthonous T. callipaeda infection in dogs, cats and foxes in the southern part of Switzerland. This area is situated at comparable latitudes $\left(46^{\circ} 00^{\prime} \mathrm{N}\right)$ to Piedmont in northern Italy and to Dordogne region in France where canine thelaziosis has previously been reported
(Rossi and Bertaglia, 1989; Dorchies et al., 2007). Cases are distributed over the southern part of the study area, at altitudes from 216 to $863 \mathrm{~m}$ a.s.l. and contiguous to Italy. Climate, altitudes and vegetation in the neighbouring Piedmont are very similar, since both are characterized by cultivated areas and deciduous woods. The presence of a potential vector, the zoophilic fruitfly $P$. variegata, which is a known vector and intermediate host of T. callipaeda in southern Italy, has been reported in southern and central Ticino (http:// www.taxodros.unizh.ch/, compiled by Bächli). This territory is also predicted to be a suitable habitat for $P$. variegata flies based on a predictive geoclimatic model (Otranto et al., 2006a). Additionally, a dog population of approximately 20,000 in the study area and an overall prevalence of $5.3 \%$ suggest that more than a thousand dogs could be infected and contribute to the expansion of thelaziosis. The particularly dense dog population in the southern part of Ticino (11,339/19,947 dogs, 56.8\%) is ideal for the establishment of an endemic area for $T$. callipaeda, assuming that a vector is also present. However, a prevalence of $6.2 \%$ in dogs in the southern part of Ticino is lower than those reported from northern (Piedmont, 23.1\%) and southern Italy (Basilicata, $41.8 \%$, Otranto et al., 2003). 


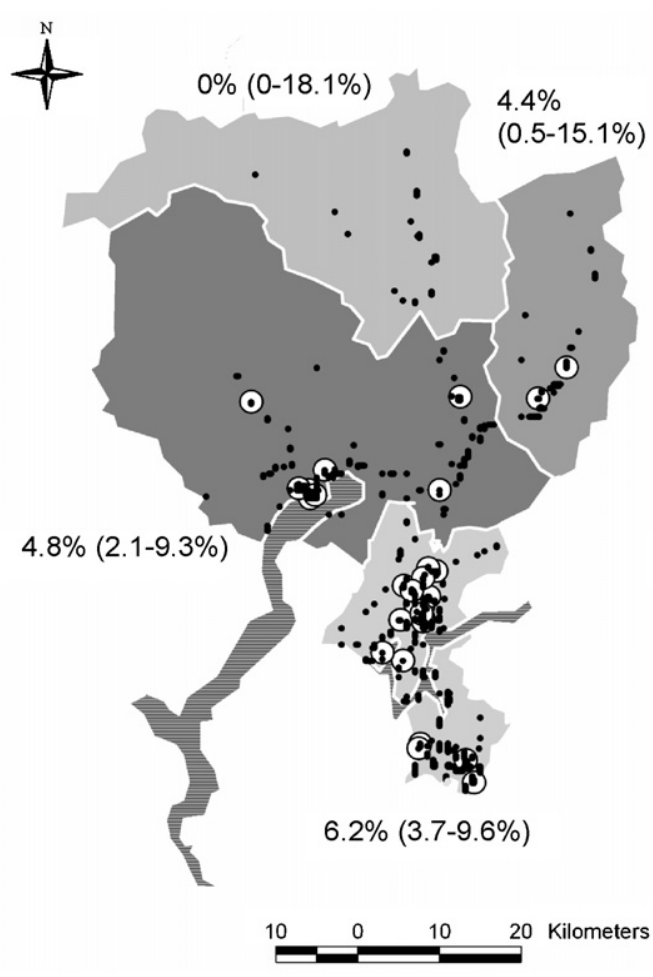

A: dogs $(n=529)$

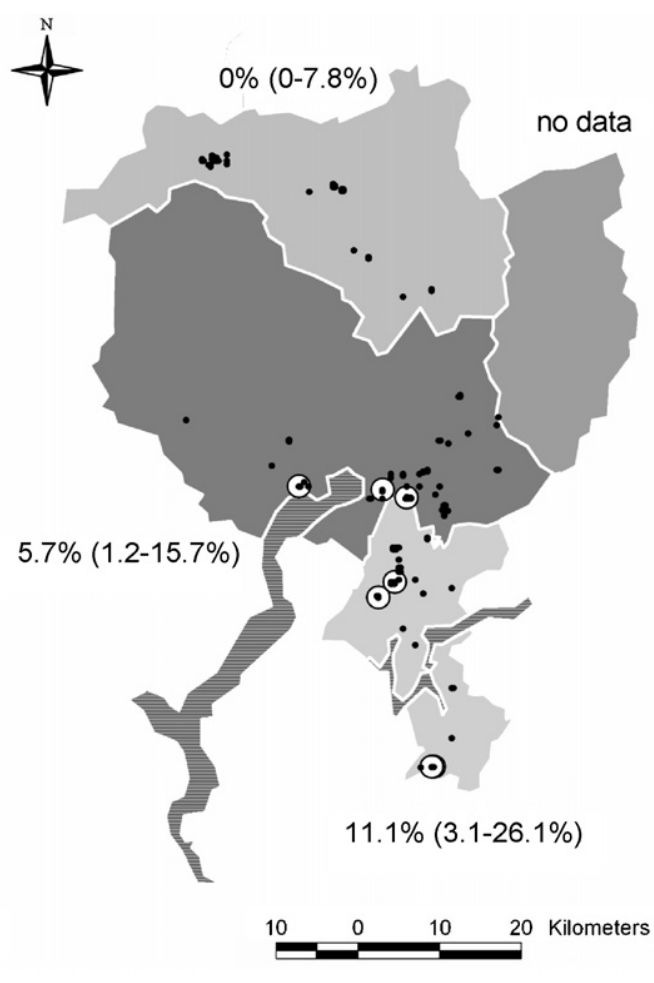

B: foxes $(n=126)$

Fig. 2. Distribution and prevalence (with $95 \%$ CI) of Thelazia callipaeda in 4 geographically subdivided parts of southern Switzerland: (A) in 28 of 529 dogs presented in veterinary clinics and (B) in 7 of 126 foxes. White dots = positive animals and black dots = negative animals.

The absence of infected animals in the northern part of Ticino may be due to the fact that the parasite has not yet spread more northwards. Alternatively, parasite transmission in the vector may be limited by climatic factors. A lower dog population (1072 dogs) and higher altitudes north of the study region, and in particular the Alps may represent a barrier to the expansion northwards. However, Thelazia-infected dogs and cats with sojourn in Ticino have already been diagnosed north of the Alps in Switzerland (Tschuor, personal communications). Furthermore, potential vectors are present in central Europe (http://www.taxodros.unizh.ch/) so that a further establishment of the parasite in Europe can be expected.

Data about gender, age and breed size of the whole dog population in Ticino, of Thelazia-positive dogs diagnosed in 2000-2007 and of dogs from the random subpopulation of the cross-sectional study are shown in Table 1. The stepwise logistic regression procedure for the data of the 529 dogs from the cross-sectional study indicated a significant final model (model $\chi^{2}=21.0$, d.f. $=3, p<0.001$ ) with a highly significant influence of the variable 'age class' (LR 11.9, d.f. $=2, p=0.003$ ) and the variable 'breed size' (LR 9.0, d.f. $=2$, $p=0.011$ ) with lower prevalence rates in young dogs and small sized breeds (Table 2). The variable 'gender' did not enter the final model. However, data from diagnosed Thelazia-positive dogs in 2000-2007 $(n=106)$ indicated a significant higher prevalence in male dogs (see CI, Table 1). In previous reports, no effect of gender, breed or age of dogs and foxes on Thelazia-infection was detected (Otranto et al., 2003). The significant lower prevalence in dogs of small breed and a low numbers of cases in cats in this study may be due to their small body mass and hence being less attractive to flies. Additionally, it is well known that cats have intensive cleaning habits usually eliminating eye discharges which are attractive to vectors. Alternatively, practitioners may have greater difficulties in inspecting cats' eyes (Otranto et al., 2003).

Of 95 Thelazia-positive dogs with known data about travel history $40(42.1 \%$ ) had previously (1 year before diagnosis) been to Italy (Table 1). The cross-sectional study showed that $44 \%$ of the dogs in Ticino have a travel history to Italy. A similar number of the Thelazianegative $\operatorname{dogs}(218 / 501,43.5 \%)$ had sojourned in Italy. Correspondingly, the factor 'sojourn in Italy' did not enter the final model of the cross-sectional study (Table 2). 
Table 1

Anamnestic data of the total dog population in Ticino, the reported Thelazia-positive dogs in 2000-2007 and the dogs from a subpopulation (cross-sectional study in July-September 2006)

\begin{tabular}{|c|c|c|c|c|c|c|c|c|c|c|c|c|c|c|}
\hline & \multicolumn{3}{|c|}{$\begin{array}{l}\text { Dog population in Ticino } \\
(n=19,947)\end{array}$} & \multicolumn{3}{|c|}{$\begin{array}{l}\text { Thelazia-positive dogs 2000- } \\
2007(n=106)\end{array}$} & \multicolumn{3}{|c|}{$\begin{array}{l}\text { Cross-sectional study } \\
\text { population }(n=529)\end{array}$} & \multicolumn{5}{|c|}{ Thelazia-positive dogs in cross-sectional study $(n=28)$} \\
\hline & \multirow[b]{2}{*}{$\mathrm{Nr}$. } & \multirow[b]{2}{*}{$\%$} & \multirow[b]{2}{*}{$95 \% \mathrm{CI}$} & \multirow[b]{2}{*}{ Nr. } & \multirow[b]{2}{*}{$\%$} & \multirow[b]{2}{*}{$95 \% \mathrm{CI}$} & \multirow[b]{2}{*}{$\mathrm{Nr}$. } & \multirow[b]{2}{*}{$\%$} & \multirow[b]{2}{*}{$95 \% \mathrm{CI}$} & \multirow[b]{2}{*}{ Nr. } & \multirow[b]{2}{*}{$\%$} & \multirow[b]{2}{*}{$95 \% \mathrm{CI}$} & \multicolumn{2}{|c|}{ Comparison $^{\mathrm{b}}$} \\
\hline & & & & & & & & & & & & & $\%$ & $95 \% \mathrm{CI}$ \\
\hline \multicolumn{15}{|l|}{ Gender } \\
\hline Male & 10,286 & 51.6 & $51.0-52.3$ & 64 & 62.1 & $52.0-71.5$ & 259 & 49.0 & $44.6-53.3$ & 15 & 53.6 & $33.9-72.5$ & 5.8 & $3.3-9.4$ \\
\hline Female & 9,630 & 48.4 & $47.7-49.0$ & 39 & 37.9 & $28.5-48.0$ & 270 & 51.0 & $46.7-55.4$ & 13 & 46.4 & $27.5-66.1$ & 4.8 & $2.6-8.1$ \\
\hline Total & $19,916^{\mathrm{a}}$ & 100.0 & & $103^{\mathrm{a}}$ & 100.0 & & 529 & 100.0 & & 28 & 100.0 & & 5.3 & $3.5-7.6$ \\
\hline \multicolumn{15}{|l|}{ Age } \\
\hline $1-3$ years & 5,238 & 26.5 & $25.9-27.1$ & 23 & 21.9 & $14.4-31.0$ & 165 & 31.2 & $27.3-35.3$ & 2 & 7.1 & $0.9-23.3$ & 1.2 & $0.1-4.3$ \\
\hline $4-8$ years & 8,510 & 43.0 & $42.3-43.7$ & 49 & 46.7 & $36.9-56.7$ & 205 & 38.7 & $34.6-43.1$ & 17 & 60.7 & $40.6-78.5$ & 8.3 & $4.9-12.9$ \\
\hline$>8$ years & 6,038 & 30.5 & $29.9-31.2$ & 33 & 31.4 & $22.7-41.2$ & 159 & 30.1 & $26.2-34.2$ & 9 & 32.1 & $15.9-52.4$ & 5.7 & $2.6-10.5$ \\
\hline Total & $19,786^{\mathrm{a}}$ & 100.0 & & $105^{\mathrm{a}}$ & 100.0 & & 529 & 100.0 & & 28 & 100.0 & & 5.3 & $3.5-7.6$ \\
\hline \multicolumn{15}{|l|}{ Breed size } \\
\hline Small & 2,520 & 48.4 & $47.1-49.8$ & 6 & 6.3 & $2.4-13.2$ & 119 & 31.7 & $27.0-36.6$ & 2 & 7.2 & $0.9-23.3$ & 1.7 & $0.2-5.9$ \\
\hline Middle & 1,267 & 24.4 & $23.2-25.5$ & 12 & 12.6 & $6.7-21.0$ & 76 & 20.2 & $16.3-24.6$ & 4 & 35.7 & $18.6-55.9$ & 5.3 & $1.5-12.9$ \\
\hline Big and very big & 1,417 & 27.2 & $26.0-28.5$ & 77 & 81.1 & $71.7-88.4$ & 181 & 48.1 & $43.0-53.3$ & 16 & 57.1 & $37.2-75.5$ & 8.8 & $5.1-14$ \\
\hline Total & $5,204^{\mathrm{a}}$ & 100.0 & & $95^{\mathrm{a}}$ & 100.0 & & $376^{\mathrm{a}}$ & 100.0 & & $22^{\mathrm{a}}$ & 100.0 & & $5.9^{\mathrm{a}}$ & $3.7-8.7$ \\
\hline \multicolumn{15}{|l|}{ Travel to Italy } \\
\hline Yes & & & & 40 & 42.1 & $32.0-52.7$ & 233 & 44.0 & $39.8-48.4$ & 12 & 42.9 & $24.5-62.8$ & 12.0 & $2.7-8.8$ \\
\hline No & & & & 55 & 57.9 & $47.3-68.0$ & 296 & 56.0 & $51.6-60.2$ & 16 & 57.1 & $37.2-75.5$ & 5.4 & $3.1-8.6$ \\
\hline Total & & & & $95^{\mathrm{a}}$ & 100 & & 529 & 100 & & 28 & 100.0 & & 5.3 & $3.5-7.6$ \\
\hline
\end{tabular}

${ }^{\text {a }}$ Information was not available for all dogs.

b Comparison of Thelazia-positive dogs with the total cross-sectional study population. 
Table 2

Odds-ratios (OR), 95\% confidence intervals (CI) and p-values of stepwise backward logistic regressions for the Thelazia-infection in dogs

\begin{tabular}{llr}
\hline Explanatory variables (test category/reference category) & Thelazia-infection in dogs $(n=529)$ & $95 \%$ CI for OR \\
\cline { 2 - 3 } & OR & $0.03-0.58$ \\
\hline Age class & 0.13 & $0.24-1.35$ \\
Young dogs vs. middle aged dogs & 0.57 & $0.01-0.78$ \\
Old dogs vs. middle aged dogs & & $0.07-1.52$ \\
Breed size & 0.10 & $0.38-2.00$ \\
Small size vs. big size & 0.33 & 0.87 \\
Medium size vs. big size & n.e. \\
Unknown size vs. big size & n.e. \\
Gender (female/male) & n.e. \\
Regional location (north/south/central Ticino) & \\
Sojourn in Italy (no/yes) & \\
\hline
\end{tabular}

n.e.: variable did not enter the final model. Age classes: $1-3$ years $=$ young, 4-8 years $=$ middle, $>8$ years $=$ old. Breed size: small $($ about $45 \mathrm{~cm}$ breast height, $<15 \mathrm{~kg}$ body weight), medium $(45-55 \mathrm{~cm}, 16-25 \mathrm{~kg})$, big $(56-70 \mathrm{~cm}, 26-45 \mathrm{~kg}$ ) and very big $(>70 \mathrm{~cm}, \mathrm{more}$ than $45 \mathrm{~kg})$.

Keratitis was diagnosed in only 4/118 dogs (3.4\%) by veterinarians, but conjunctivitis in $96 / 118$ dogs (81.4\%), while $78 / 118$ dogs $(66.1 \%)$ showed epiphora. Thelaziosis in cats was diagnosed in four males and one animal of unknown gender. From this latter cat, the data record was incomplete. None of the four other cats showed epiphora, but two showed conjunctivitis and the two other cats had keratitis, even though they were harbouring low worm numbers. Two males and three female worms were found in the infected cats (i.e., one per eye in three cats and two in a single eye of a cat). Cats were 6, 7, 8 and 11 years old and belonged to the European short-hair breed. They never have been abroad.

In foxes, the number of worms per eye varied from one to ten, with five foxes having $T$. callipaeda in both eyes. Two of the positive foxes were females, while the other five positive foxes were males.

From totally 112 Thelazia-positive dogs, including dogs from the cross-sectional study, it was known which eyes were infected. Thirty-four $(30.4 \%, 95 \%$ $\mathrm{CI}=22.0-39.8)$ housed eye worms in their right eye only, $42(37.5 \%, 95 \% \mathrm{CI}=28.5-47.1)$ in the left and 36 $(32.1 \%, 95 \% \mathrm{CI}=23.6-41.6)$ in both eyes, showing that there are no significant differences. The number of worms per eye varied between 1 and 23. Of the $63 \mathrm{dogs}$ for which complete data about nematode identification were available, $10 \quad(15.9 \%, 95 \% \quad \mathrm{CI}=7.9-23.7)$ harboured male nematodes and $26(41.3 \%, 95 \%$ CI $=29.0-54.4)$ female $T$. callipaeda . Dogs harboured therefore significantly more female than male nematodes $(p=0.0028)$. Only 27 dogs $(42.8 \%, 95 \%$ $\mathrm{CI}=30.5-56.0)$ had worms of both gender. This latter observation as well as the fact that several animals harboured only one worm per eye indicates that not all infected animals may contribute to parasite transmission and that infection pressure for vectors is low. Low worm numbers may cause mild clinical symptoms and pass unobserved by the owner and by veterinarians. Additionally, epiphora and conjunctivitis can have other aetiologies. Therefore, particularly at the beginning of the study, some of such cases may not have been attributed to thelaziosis. Underdiagnosis can therefore also be a cause of lower prevalences. However, this was not the case in our cross-sectional study.

Since data were collected for this study, the number of diagnosed cases is rapidly increasing, in particular in southern Ticino (Nägeli, unpublished). A wild animal cycle in foxes is probable because they are abundant in the region, have high migration activity, are often urban dwellers with close contact to domestic animals and also harbour high worm numbers.

\section{Acknowledgements}

The authors thank the veterinarians Athos Binda, Jürg Eitel, Alberto Stern, Davide Lafranchi, Daniele Varini, Andreas Dürr, Marco Manetti, Paola Juri, Wolfgang Rohr, Andrea Martina, Roberto Mossi, Michele Mazzi, Martina Casella and the veterinarians from the Centro Veterinario Keller \& Pedretti for the precious collaboration finding cases.

This work represents the dissertation of Filippo Malacrida, veterinarian.

\section{References}

Anderson, R.C., 2000. Nematode parasites of vertebrates: their development and transmission, second ed. CABI Publishing, Guilford, UK, pp. 404-407. 
Bhaibulaya, M., Prasertsilpa, S., Vajrasthira, S., 1970. Thelazia callipaeda Railliet and Henry, 1910, in man and dog in Thailand. Am. J. Trop. Med. Hyg. 19, 476-479.

Dorchies, P., Chaudieu, G., Siméon, L.A., Cazalot, G., Cantacessi, C., Otranto, D., 2007. First reports of autochthonous eyeworm infection by Thelazia callipaeda (Spirurida, Thelaziidae) in dogs and cat from France. Vet. Parasitol. 149, 294297.

Norusis, M.J., 1986. SPSS/PC+ advanced statistics. SPSS Marketing Department, Chicago.

Otranto, D., Traversa, D., 2005. Thelazia eyeworm: an original endoand ecto-parasitic nematode. Trends Parasitol. 21, 1-4.

Otranto, D., Dutto, M., 2008. Human thelaziosis, Europe. Emerg. Infect. Dis. 14, 647-649.

Otranto, D., Ferroglio, E., Lia, R.P., Traversa, D., Rossi, L., 2003. Current status and epidemiological observation of Thelazia callipaeda (Spirurida, Thelaziidae) in dogs, cats and foxes in Italy: a "coincidence" or a parasitic disease of the Old Continent? Vet. Parasitol. 116, 315-325.
Otranto, D., Lia, R.P., Cantacessi, C., Testini, G., Troccoli, A., Shen, J.L., Wang, Z.X., 2005a. Nematode biology and larval development of Thelazia callipaeda (Spirurida, Thelaziidae) in the drosophilid intermediate host in Europe and China. Parasitology 131, 847-855.

Otranto, D., Testini, G., De Luca, F., Hu, M., Shamsi, S., Gasser, R.B., 2005b. Analysis of genetic variability within Thelazia callipaeda (Nematoda: Thelazioidea) from Europe and Asia by sequencing and mutation scanning of the mitochondrial cytochrome $c$ oxidase subunit 1 gene. Mol. Cell Probes 19, 306-313.

Otranto, D., Brianti, E., Cantacessi, C., Lia, R.P., Màca, J., 2006a. The zoophilic fruitfly Phortica variegata: morphology, ecology and biological niche. Med. Vet. Entomol. 20, 358-364.

Otranto, D., Cantacessi, C., Testini, G., Lia, R.P., 2006b. Phortica variegata as an intermediate host of Thelazia callipaeda under natural conditions: evidence for pathogen transmission by a male arthropod vector. Int. J. Parasitol. 36, 1167-1173.

Rossi, L., Bertaglia, P.P., 1989. Presence of Thelazia callipaeda Railliet \& Henry, 1910 in Piedmont, Italy. Parassitologia 31, 167-172. 